\title{
Increased Serum Cystatin C Levels Were Associated with Depressive Symptoms in Patients with Type 2 Diabetes
}

This article was published in the following Dove Press journal: Diabetes, Metabolic Syndrome and Obesity: Targets and Therapy

\author{
Yue Huang ${ }^{1} *$ \\ Wenxun Huang ${ }^{2, *}$ \\ Jing $\mathrm{Wei}^{3}$ \\ Zubin Yin' \\ Hanjing Liu ${ }^{4}$ \\ 'Department of Psychosomatic Medicine, \\ Chongqing University Three Gorges \\ Hospital, Chongqing, 404000, People's \\ Republic of China; ${ }^{2}$ Department of \\ Infectious Disease, Chongqing University \\ Three Gorges Hospital, Chongqing, \\ 404000, People's Republic of China; \\ ${ }^{3}$ Department of Endocrinology, \\ Chongqing Municipality Wanzhou \\ District People's Hospital, Chongqing, \\ 404000, People's Republic of China; \\ ${ }^{4}$ Department of Clinical Psychology, The \\ Chinese People's Armed Police Force \\ Zhejiang Corps Hospital, Hangzhou, \\ 3I005I, Zhejiang Province, People's \\ Republic of China
}

*These authors contributed equally to this work
Correspondence: Hanjing Liu Department of Clinical Psychology, The Chinese People's Armed Police Force

Zhejiang Corps Hospital, No. 86, Jiangnan Avenue, Hangzhou, 31005I, Zhejiang

Province, People's Republic of China Tel +86 I387445I742

Email liuhanjing666@I26.com
Background: Existing studies have reported that patients with diabetes mellitus (DM) have an increased risk of depressive symptoms. We aimed to evaluate the association between serum cystatin $\mathrm{C}$ levels and depressive symptoms in DM patients.

Methods: Serum levels of cystatin C were measured in 254 patients with DM at baseline. Cox proportional hazard analysis was used to evaluate the value of serum cystatin $\mathrm{C}$ in predicting depressive symptoms in patients with DM.

Results: Multivariate linear regression analysis showed that serum cystatin $\mathrm{C}$ levels were independently associated with Centre for Epidemiological Studies Depression (CES-D) scores after adjusting for age, sex, body mass index (BMI), current smoking status, current drinking, admission systolic and diastolic blood pressure (BP), cardiovascular disease (CVD) history and laboratory measurements in patients with $\mathrm{DM}$ at baseline $(\mathrm{S} \beta=-0.127 ; 95 \% \mathrm{CI}$, 0.185 - $0.083 ; P=0.002)$. The multivariate Cox proportional hazard analysis revealed that serum cystatin $\mathrm{C}(\mathrm{HR}=2.360,95 \%$ CI $1.500-3.891, P$-trend $<0.001)$ was an independent prognostic factor for cognitive decline in these patients with DM during the follow-up period.

Conclusion: Our results showed that increased serum cystatin $\mathrm{C}$ levels were significantly and independently associated with depressive symptoms and had independent predictive value for depressive symptoms in patients with DM. Serum cystatin C might enable early recognition of depressive symptoms among DM patients.

Keywords: cystatin C, DM, depressive symptoms, prognostic value

\section{Introduction}

Diabetes mellitus (DM) has been strongly associated with an increased risk of developing depressive symptoms. ${ }^{1-3}$ Existing studies have suggested that the risk of depression is higher in people with DM than in those without DM. ${ }^{4-6}$ Although the current mechanisms are poorly understood, increased inflammation and oxidative stress in DM play important roles in an increased risk of depression. ${ }^{7-9}$ Depressive symptoms are a major health problem and have been identified as strong prognostic factors of heavy medical burden and poor quality of life in patients with DM. ${ }^{10}$ Therefore, identifying relevant risk factors for depressive symptoms in patients with DM must be addressed.

Cystatin $\mathrm{C}$ is a low-molecular-weight protein $(13.4 \mathrm{kDa})$ that is synthesized at a constant rate in all nucleated cells. ${ }^{11}$ Cystatin $\mathrm{C}$ is freely filtered through the glomerulus and then reabsorbed and fully catabolized without secretion or 
subsequent reabsorption into the circulation. ${ }^{12}$ Serum cystatin $\mathrm{C}$ is less likely to be influenced by individual characteristics, such as age, sex, ethnicity, muscle mass, and medication use, than serum creatinine. ${ }^{13-15}$ thus, researchers have proposed cystatin $\mathrm{C}$ as a more reliable endogenous marker of early renal dysfunction. ${ }^{15,16}$ However, the results of previous studies have also suggested that serum cystatin $\mathrm{C}$ may be an independent risk factor for all-cause mortality among elderly persons with cardiovascular diseases (CVDs). ${ }^{17-19}$ It has also been reported that higher serum cystatin $\mathrm{C}$ may be associated with an increased risk of CVDs, such as stroke and coronary heart disease, and subsequent mortality, even in individuals without established chronic kidney disease (CKD) ${ }^{13-15,19}$ The aim of our study was to evaluate whether serum cystatin $\mathrm{C}$ levels are an independent predictor of depressive symptoms in patients with DM.

To date, few studies have explored the association between serum cystatin $\mathrm{C}$ levels and depression in patients with DM. The aim of this study was to investigate whether increased serum levels of cystatin $\mathrm{C}$ contribute to an increased risk of depressive symptoms. This was also the first study to explore the prognostic value of serum cystatin $\mathrm{C}$ for predicting depression in patients with DM.

\section{Materials and Methods Study Population}

We studied 254 hospitalized patients who were admitted to Chongqing University Three Gorges Hospital and the Chinese People's Armed Police Force Zhejiang Corps Hospital in China because of DM between January 2013 and December 2015. All eligible DM patients had no other serious acute illnesses, including acute dysfunction of liver or kidney function or acute heart failure, and they and were stable for more than three months before admission. There are three diagnostic criteria for diabetes: typical symptoms of diabetes, such as polydipsia, polydipsia, polyuria and emaciation, or a random serum glucose level greater than or equal to $11.1 \mathrm{mmol} / \mathrm{l}$; a fasting serum glucose level greater than or equal to $7.0 \mathrm{mmol} / \mathrm{L}$; and blood glucose and serum glucose levels greater than or equal to $11.1 \mathrm{mmol} / \mathrm{L}$ two hours after glucose loading. The above diagnostic criteria must be repeated on different days before diagnosis. ${ }^{20}$ The diagnosis of DM in the current study was performed by two endocrinologists who used the same diagnostic criteria for the included DM patients. Patients with DM were clinically stable during hospitalization and were prospectively followed up after discharge. Of these patients, 53 patients with DM were identified as having depressive symptoms in this study. Patients $(\mathrm{N}=19)$ with liver dysfunction, kidney dysfunction, neoplastic diseases, or other serious diseases were excluded.

The diagnosis of CVDs, including coronary heart disease, stroke, hypertension and others, was based on the patient's current or previous medical records. Other data on clinical characteristics, including age, sex, body mass index (BMI), smoking, alcohol consumption, blood pressure (BP) and others, were collected from patient interviews or medical records. According to the Declaration of Helsinki guidelines, the Ethics Committee of Chongqing University Three Gorges Hospital and the Chinese People's Armed Police Force Zhejiang Corps Hospital approved this study, and all patients gave written informed consent.

\section{Blood Specimen Collection and Laboratory Analysis}

Venous blood samples from patients with DM were collected within the first 24 hours after admission. The samples were prepared immediately by centrifugation and processed for determination of cystatin C. Serum levels of cystatin $\mathrm{C}$ were measured via particle-enhanced immunonephelometric assay using the Siemens ADVIA 2400 autoanalyzer (Siemens AG, Munich, Germany). Fasting venous blood samples from patients with DM were also tested for albumin (ALB), blood glucose (FBG), hemoglobin $(\mathrm{Hb}$ ), fasting hs-CRP (hs-C reactive protein), HbAlc (glycosylated hemoglobin), high density lipoprotein (HDL) and LDL (low density lipoprotein) with the use of the Siemens ADVIA 2400 automatic biochemistry analyzer (Siemens AG). The estimated glomerular filtration rate (eGFR) was calculated by using the Chronic Kidney Disease Epidemiology Collaboration Group (CKD-EPI) equation. $^{21}$

\section{Depression Score}

The Centre for Epidemiological Studies Depression (CESD) scale was used to calculate depressive scores. ${ }^{22,23}$ A cutoff score of 16 was considered indicative of depressive symptoms at baseline. During the follow-up period, a score $\geq 16$ was considered indicative of depression events. The CES-D scale has been used widely in epidemiological studies and is appropriate for use in our 
studies. ${ }^{22,23}$ In addition, the subjects were considered to have depression if they took antidepressant medications.

\section{Follow-Up}

All patients were followed prospectively after discharge for a median period of 35 months (range $=3-37$ ). Patients were followed by telephone or review of the medical record four times a year until the occurrence of depressive symptoms (depression event).

\section{Statistical Analyses}

The Kolmogorov-Smirnov test was used to analyze the normality of the data. A $P$ value $\leq 0.05$ was considered to be statistically significant. The data that were not normally distributed were analyzed by the Mann-Whitney U-test and then expressed as the median (interquartile range [IQR]). Data were analyzed by independent $t$-test and presented as the mean \pm SD for normally distributed data. The chi-square test was used to analyze categorical variables and is expressed as $\mathrm{n}(\%)$. A multivariate linear regression model was used to assess the independent relationship between serum cystatin $\mathrm{C}$ levels and CES-D scores in DM patients at baseline after controlling for age, sex, BMI, current smoking status, current alcohol consumption status, admission systolic and diastolic BP, CVD history and laboratory measurements. Then, Cox proportional hazard analysis was performed to assess the independent prognostic factors for depressive symptoms in patients with DM during the followup period after controlling for these same confounding factors. In these multivariate analyses, we adjusted for clinical data relevant to stroke and cognitive function even if the factors were not significantly associated with depressive symptoms in the univariate analysis because they are key clinical variables and may be associated with depressive symptoms in the multivariate but not univariate analyses. Additionally, sensitivity and stratification analyses were also performed to evaluate the independent association between serum cystatin $\mathrm{C}$ levels and depressive symptoms (depression event) by adding "antidepressant therapy" and "CKD history" as covariates. All of the analyses were performed by using SPSS 24.0.

\section{Results}

\section{Clinical Characteristics of Study Subjects}

\section{at Baseline}

The clinical characteristics of the DM patients at baseline are presented in Table 1. The mean age of all included DM
Table I Clinical Characteristics in 254 Patients with DM at Baseline

\begin{tabular}{|c|c|}
\hline Variables & All Patients with DM $(n=254)$ \\
\hline Age (years) & $63.2 \pm 7.9$ \\
\hline Gender (male), n (\%) & $120(47.2)$ \\
\hline $\mathrm{BMI}(\mathrm{kg} / \mathrm{m} 2)$ & $25.6 \pm 5.3$ \\
\hline Current smoker, n (\%) & $72(28.3)$ \\
\hline Current drinker, n (\%) & $87(34.3)$ \\
\hline Admission systolic BP ( $\mathrm{mmHg})$ & |38.| (|32.6-|47.8) \\
\hline Admission diastolic BP $(\mathrm{mmHg})$ & $85.6(80.3-95.3)$ \\
\hline Antidepressant therapy, $\mathrm{n}(\%)$ & $31(9.6)$ \\
\hline CESD score & $10.7 \pm 9.2$ \\
\hline Depressive symptoms, n (\%) & $53(20.9)$ \\
\hline \multicolumn{2}{|l|}{ CVD history } \\
\hline Hypertension, n (\%) & $135(53.1)$ \\
\hline Coronary heart disease, $\mathrm{n}(\%)$ & $31(12.2)$ \\
\hline Stroke, n (\%) & $24(9.4)$ \\
\hline CKD history & II (43.3) \\
\hline \multicolumn{2}{|l|}{ Laboratory measurements } \\
\hline eGFR $\left(\mathrm{mL} / \mathrm{min} / 1.73 \mathrm{~m}^{2}\right)$ & $62.5(57.6-65.3)$ \\
\hline $\mathrm{Hs}-\mathrm{CRP}(\mathrm{pg} / \mathrm{mL})$ & $8.3(5.1-15.3)$ \\
\hline $\mathrm{Hb}(\mathrm{g} / \mathrm{L})$ & $108.4 \pm 4.6$ \\
\hline $\operatorname{ALB}(g / L)$ & $34.5 \pm 3.9$ \\
\hline FBG (mmol/L) & $12.8 \pm 2.9$ \\
\hline $\mathrm{HbAlc}(\%)$ & $10.1(8.1-12.4)$ \\
\hline $\mathrm{HDL}(\mathrm{mmol} / \mathrm{L})$ & $1.3 \pm 0.2$ \\
\hline LDL (mmol/L) & $2.7 \pm 1.1$ \\
\hline Serum cystatin C (mg/L) & $1.39(1.03-1.52)$ \\
\hline
\end{tabular}

Note: Data are presented as mean \pm SD for normally distributed data, as median (interquartile range) for nonnormally distributed data, and as $\mathrm{n}$ (\%) for categoric variables.

Abbreviations: DM, diabetes mellitus; BMI, body mass index; BP, blood pressure; CESD, Centre for Epidemiological Studies Depression; CVD, cardiovascular disease; CKD, chronic kidney disease; eGFR, estimated glomerular filtration rate; $\mathrm{Hb}$, hemoglobin; hs-CRP, hs-C-reactive protein; ALB, albumin; FBG, fasting blood glucose on admission; HbAlc, glycosylated hemoglobin; HDL, high-density lipoprotein; LDL, low-density lipoprotein.

patients was $63.2 \pm 7.9$ years, and there were 120 males (47.2\%). The CES-D score was $10.7 \pm 9.2$, and the total number of patients with depressive symptoms was 53 (20.9\%). The median value of serum cystatin $\mathrm{C}$ levels was $1.39(\mathrm{mg} / \mathrm{L})$.

\section{Increased Serum Levels of Cystatin C Showed a Significant Association with CES-D Score in 254 Patients with DM}

To assess the association of serum cystatin $\mathrm{C}$ levels in stroke patients with CES-D scores, a multivariate linear regression model was performed (Table 2). Model 1 showed that higher serum levels of cystatin $\mathrm{C}$ were 
Table 2 Multivariate Linear Regression Analysis for the Association Between the Serum Cystatin C Levels and CESD Score in 254 Patients with DM at Baseline

\begin{tabular}{|l|c|c|c|c|}
\hline Variables & $\mathbf{R}^{\mathbf{2}}$ & $\mathbf{S \beta}$ & $\mathbf{9 5 \%} \mathbf{C l}$ & $\mathbf{P}$ value \\
\hline $\begin{array}{l}\text { Serum cystatin C (mg/ } \\
\text { L) (per I-SD increase) }\end{array}$ & & & & \\
\hline Model I & 0.124 & 0.153 & $0.104-0.224$ & $<0.001$ \\
Model 2 & 0.153 & 0.143 & $0.095-0.200$ & $<0.001$ \\
Model 3 & 0.196 & 0.127 & $0.083-0.185-$ & 0.002 \\
\hline
\end{tabular}

Notes: Model I: No adjustment Model 2: Adjusted for age, gender, BMI, current smoker, current drinker, admission systolic and diastolic BP and CVD history. Model 3: Adjusted for age, gender, BMI, current smoker, current drinker, admission systolic and diastolic BP, CVD history and laboratory measurements.

Abbreviations: DM, diabetes mellitus; CESD, Centre for Epidemiological Studies Depression; BMI, body mass index; BP, blood pressure; CVD, cardiovascular disease.

significantly associated with CES-D score after no adjustment was made $(\mathrm{S} \beta=0.153,95 \%$ CI $0.104-0.224$, $\mathrm{P}<0.001)$. The results of Model 2 were similar to those of Model 1 ( $\mathrm{S} \beta=0.143$, 95\% CI $0.095-0.200, \mathrm{P}<0.001)$ after adjustments were made for age, sex, BMI, current smoking status, current drinking status, admission systolic and diastolic BP and CVD history. This relationship in Model 3 remained statistically significant and changed minimally after adding laboratory measurements to Model 2 ( $\mathrm{S} \beta=0.127$, 95\% CI 0.083-0.185, $\mathrm{P}=0.002)$.

\section{Cox Proportional Hazard Analysis for Predicting Depressive Symptoms (Depression Events) in 254 DM Patients}

All included DM patients $(\mathrm{N}=254)$ were prospectively followed up for a median period of 35 months (range=3-37). Depressive symptoms (depression events) occurred in 53 of the included DM patients. To assess the risk factors for depression events, a multivariate Cox proportional hazard regression model was used (Table 3). After adjustments were made for age, sex, BMI, current smoking status, current drinking status, admission systolic and diastolic BP, CVD history and laboratory measurements, multivariate Cox proportional hazard analysis suggested that serum cystatin C levels $\quad(\mathrm{HR}=2.360$, 95\% CI 1.500-3.891, $P$-trend $<0.001)$ were an independent prognostic factor for depression events. Kaplan-Meier analysis showed that DM patients with serum levels of cystatin $\mathrm{C}$ above the median had a significantly higher rate of depression events than patients with serum levels of cystatin $\mathrm{C}$ below the median value (Log rank test, $P<0.001$, Figure 1).

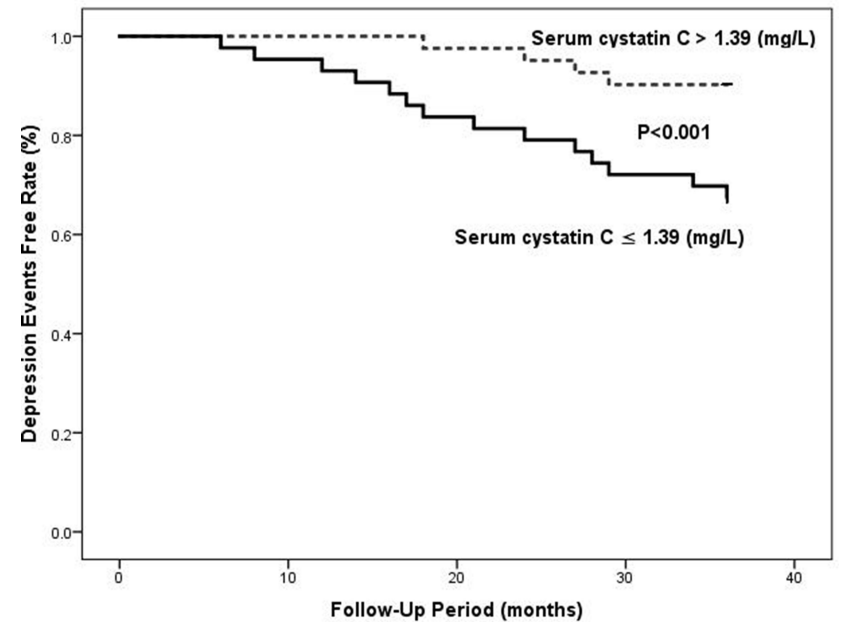

Figure I Kaplan-Meier analysis of depression events free stratified into 2 groups by the median levels of serum cystatin $C$.

\section{Sensitivity and Stratification Analysis for Predicting Depressive Symptoms (Depression Events) in 254 DM Patients}

We performed an additional sensitivity analysis to evaluate the association of serum cystatin $\mathrm{C}$ with depression events in DM patients by adding "antidepressant therapy" as a covariate (Table 4). The sensitivity analysis suggested that higher serum cystatin $\mathrm{C}$ levels were still independently associated with a higher risk of depression events during the follow-up of 3 years. Stratified analysis, performed by adding "CKD history" as a covariate, showed that the significant association between serum cystatin $C$ levels and depression events in DM patients was affected by CKD history. A significant association existed in patients with a history of CKD but not in patients without a history of CKD (Table 5).

\section{Discussion}

In this study, we investigated whether serum cystatin $\mathrm{C}$ levels can serve as an independent predictive marker for the prognosis of depression events in patients with DM. Our results suggested that serum cystatin $\mathrm{C}$ levels were an independent prognostic factor for depression events in DM patients by multivariate Cox proportional hazard analysis.

It is currently unclear whether cystatin $\mathrm{C}$ has a close association with depression in patients with DM. Inflammation plays an important role in the pathogenesis and prognosis of DM and depression. ${ }^{24-26}$ The results of our study and other studies have suggested a close correlation between cystatin $\mathrm{C}$ and conventional inflammatory markers, 
Table 3 Cox Proportional Hazard Analysis for the Association of Serum Cystatin C Levels and Depressive Symptoms

\begin{tabular}{|l|c|c|c|}
\hline Variables & Model I & Model 2 & Model 3 \\
\hline Serum cystatin C (mg/L) (per I-SD increase) & & & \\
\hline Quartile I & 1.000 (ref.) & 1.000 (ref.) & 1.000 (ref.) \\
Quartile 2 & $1.650(1.24 I-3.319)$ & $1.540(1.217-3.115)$ & $1.462(1.200-2.813)$ \\
Quartile 3 & $2.329(1.562-3.993)$ & $2.148(1.456-3.495)$ & $1.832(1.320-3.391)$ \\
Quartile 4 & $2.738(1.773-4.405)$ & $2.684(1.598-4.123)$ & $2.360(1.500-3.891)$ \\
P-trend & $<0.001$ & $<0.001$ & $<0.001$ \\
\hline
\end{tabular}

Notes: Model I: No adjustment. Model 2: Adjusted for age, gender, BMI, current smoker, current drinker, admission systolic and diastolic BP and CVD history. Model 3: Adjusted for age, gender, BMI, current smoker, current drinker, admission systolic and diastolic BP, CVD history and laboratory measurements.

Abbreviations: DM, diabetes mellitus; CESD, Centre for Epidemiological Studies Depression; BMI, body mass index; BP, blood pressure; CVD, cardiovascular disease.

such as hs-CRP. ${ }^{27-29}$ Therefore, it is reasonable to suggest that the cystatin $\mathrm{C}$ level may be of value in predicting depressive symptoms by reflecting the amount of systemic inflammation. Indeed, examination of the relationship between serum cystatin $\mathrm{C}$ and frequent inflammatory responses in patients with DM has been reported in the literature, ${ }^{30-32}$ which is consistent with our results. In our study, we first showed that patients with higher serum levels of cystatin $\mathrm{C}$ had an increased CES-D score at baseline and then had a higher risk of depression events after a follow-up of 3 years, which may be mostly or partly explained by the research on inflammatory mechanisms performed in previous studies. ${ }^{28,30}$ These studies have suggested that chronic vascular inflammation caused by DM promotes the

Table 4 Sensitivity Analysis for the Association of Serum Cystatin C Levels and Depressive Symptoms

\begin{tabular}{|c|c|c|c|}
\hline Variables & Model I & Model 2 & Model 3 \\
\hline \multicolumn{4}{|c|}{ Serum cystatin C (mg/L) (per I-SD increase) } \\
\hline Quartile I & 1.000 (ref.) & 1.000 (ref.) & 1.000 (ref.) \\
\hline Quartile 2 & $1.631(1.320-3.211)$ & $1.565(1.28 I-3.00 I)$ & $1.501(1.201-2.991)$ \\
\hline Quartile 3 & $2.161(1.439-3.518)$ & $2.120(1.328-3.204)$ & $2.004(1.298-3.092)$ \\
\hline Quartile 4 & $2.337(1.640-4.343)$ & $2.201(1.600-4.112)$ & $2.102(1.473-3.685)$ \\
\hline$P$-trend & $<0.001$ & 0.002 & 0.009 \\
\hline
\end{tabular}

Notes: Model I: Antidepressant therapy. Model 2: Adjusted for age, gender, BMI, current smoker, current drinker, admission systolic and diastolic BP, CVD history and antidepressant therapy Model 3: Adjusted for age, gender, BMI, current smoker, current drinker, admission systolic and diastolic BP, CVD history, laboratory measurements and antidepressant therapy.

Abbreviations: DM, diabetes mellitus; CESD, Centre for Epidemiological Studies Depression; BMI, body mass index; BP, blood pressure; CVD, cardiovascular disease.

Table 5 Stratified Analysis for the Association of Serum Cystatin C Levels and Depressive Symptoms

\begin{tabular}{|c|c|c|c|}
\hline Variables & Model I & Model 2 & Model 3 \\
\hline \multicolumn{4}{|l|}{ CKD history (yes) } \\
\hline $\begin{array}{l}\text { Serum cystatin } C(\mathrm{mg} / \mathrm{L}) \text { (per I-SD increase) } \\
P \text { value }\end{array}$ & $\begin{aligned} 2.214 & (1.628-3.662) \\
& <0.001\end{aligned}$ & $\begin{aligned} 2.109 & (1.546-3.30 \mathrm{I}) \\
& <0.00 \mathrm{I}\end{aligned}$ & $\begin{aligned} 1.988 & (1.458-3.074) \\
& <0.001\end{aligned}$ \\
\hline \multicolumn{4}{|l|}{ CKD history (no) } \\
\hline $\begin{array}{l}\text { Serum cystatin } C(\mathrm{mg} / \mathrm{L}) \text { (per I-SD increase) } \\
P \text { value }\end{array}$ & $\begin{array}{c}1.621(1.202-2.335) \\
0.031\end{array}$ & $\begin{array}{c}1.4092(0.993-1.944) \\
0.076\end{array}$ & $\begin{array}{c}1.273(0.917-1.592) \\
0.112\end{array}$ \\
\hline
\end{tabular}

Notes: Model I: No adjustment Model 2: Adjusted for age, gender, BMI, current smoker, current drinker, admission systolic and diastolic BP and CVD history. Model 3: Adjusted for age, gender, BMI, current smoker, current drinker, admission systolic and diastolic BP, CVD history and laboratory measurements.

Abbreviations: DM, diabetes mellitus; CESD, Centre for Epidemiological Studies Depression; BMI, body mass index; BP, blood pressure; CVD, cardiovascular disease. 
occurrence of depression events. ${ }^{33-36}$ The early detection of depressive symptoms in these DM patients may provide us with a chance to develop strategies aiming to reduce the medical burden and improve prognosis. Our results seemed to imply that serum cystatin $\mathrm{C}$ might be a sensitive biomarker for the early recognition of depressive symptoms in patients with DM.

Our results have several obvious strengths. First, we are the first to find that serum cystatin $\mathrm{C}$ can be considered a valuable prognostic factor for depressive symptoms in DM patients. Second, we ensured comprehensive follow-up and rigorous adjudication of depression events, so our results are very reliable. Third, the serum cystatin $\mathrm{C}$ assay chosen for this study is both widely available and analytically stable (coefficient of variation of precision $<5 \%$ ). This serum index is convenient and reliable for clinical popularization.

Certainly, this study also has several limitations. The study sample was recruited from a single-center, and the sample size was fairly small. Additionally, cystatin $\mathrm{C}$ was only measured once in the morning of the first day after admission. The time courses of cystatin $\mathrm{C}$ levels were not evaluated. Information regarding the long-term prognosis throughout the hospitalization period was not available. Further investigation will be necessary to solidify the clinical significance of cystatin $\mathrm{C}$ in predicting depressive symptoms in patients with DM by evaluating the time course of cystatin $\mathrm{C}$.

\section{Conclusions}

We demonstrated that serum cystatin $\mathrm{C}$ is an independent prognostic factor for depressive symptoms in patients with DM. These results suggest that serum cystatin $\mathrm{C}$ might be a useful biomarker for predicting depressive symptoms in patients with DM.

\section{Funding}

There is no funding to report.

\section{Disclosure}

The authors report no conflicts of interest in this work.

\section{References}

1. Cezaretto A, Ferreira SRG, Sharma S, Sadeghirad B, Kolahdooz F. Impact of lifestyle interventions on depressive symptoms in individuals at-risk of, or with, type 2 diabetes mellitus: a systematic review and meta-analysis of randomized controlled trials. Nutrition Metab Cardiovasc Diseases Nmcd. 2016;26(8):649-662.

2. Lee S, Chiu A, Tsang A, et al. Treatment-related stresses and anxiety-depressive symptoms among Chinese outpatients with type 2 diabetes mellitus in Hong Kong. Diabetes Res Clin Pract. 2006;74 (3):282-288. doi:10.1016/j.diabres.2006.03.026
3. Feltz-Cornelis CMVD, Nuyen J, Stoop C, et al. Effect of interventions for major depressive disorder and significant depressive symptoms in patients with diabetes mellitus: a systematic review and meta-analysis. Gen Hosp Psychiatry. 2010;32(4):380-395. doi:10.1016/j.genhosppsych.2010.03.011

4. Salomé GM, Blanes L, Ferreira LM. Avaliao de sintomas depressivos em pessoas com diabetes mellitus e pé ulcerado. [Assessment of depressive symptoms in people with diabetes mellitus and foot ulcer]. Revista Do Colégio Brasilro De Cirurgies. 2011;38(5):327. doi:10.1590/S0100-69912011000500008. Portugese.

5. Hasan SS, Clavarino AM, Mamun AA, et al. Incidence and risk of diabetes mellitus associated with depressive symptoms in adults: evidence from longitudinal studies. Diab Metab Syndrome Clin Res Rev. 2014;8(2):82-87. doi:10.1016/j.dsx.2014.04.023

6. Mukherjee N, Chaturvedi SK. Depressive symptoms and disorders in type 2 diabetes mellitus. Curr Opin Psychiatry. 2019;32(5):416-421. doi:10.1097/YCO.0000000000000528

7. Kobrosly R, Van WE. Associations between immunologic, inflammatory, and oxidative stress markers with severity of depressive symptoms: an analysis of the 2005-2006 National health and nutrition examination survey. Neurotoxicology. 2010;31(1):126-133. doi:10.1016/j.neuro.2009.10.005

8. Maes LM. Mechanistic explanations how cell-mediated immune activation, inflammation and oxidative and nitrosative stress pathways and their sequels and concomitants play a role in the pathophysiology of unipolar depression. Neurosci Biobehav Rev. 2012.

9. Mazereeuw G, Herrmann N, Xu H, et al. Platelet activating factors are associated with depressive symptoms in coronary artery disease patients: a hypothesis-generating study. Neuropsychiatr Dis Treat. 2015;11:2309-2314.

10. Jaser SS, Holl MG, Jefferson V, et al. Correlates of depressive symptoms in urban youth at risk for type 2 diabetes mellitus. $J$ School Health. 2009. doi:10.1111/j.1746-1561.2009.00411.x

11. Okizaki SI, Moriya T, Cystatin C. Nippon rinsho. Japanese J Clin Med. 2010;9(16):419-425.

12. Inker LA, Schmid CH, Tighiouart H, et al. Estimating glomerular filtration rate from serum creatinine and cystatin C. $N$ Engl J Med. 2012;367(1):20. doi:10.1056/NEJMoa1114248

13. Anders G, Ulf N, Jonas B, et al. Simple Cystatin C-Based prediction equations for glomerular filtration rate compared with the modification of diet in renal disease prediction equation for adults and the schwartz and the counahan-barratt prediction equations for children. Clin Chem. 2005;51(8):1420-1431. doi:10.1373/clinchem.2005.051 557

14. Albert MA, Rifai N, Ridker PM. Plasma Concentration of Cystatin-C and mannose binding protein and the risk of developing peripheral vascular disease. Circulation. 2001;103(9):1351.

15. Filler G, B?Kenkamp A, Hofmann W, et al. Cystatin C as a marker of GFR-history, indications and future research. Clin Biochem. 2005;38 (1):1-8. doi:10.1016/j.clinbiochem.2004.09.025

16. Shlipak MG, Matsushita K, Johan, et al. Cystatin C versus creatinine in determining risk based on kidney function. New England J Med. 2013;369(10):932-943. doi:10.1056/NEJMoa1214234

17. O'Seaghdha CM, Tin A, Yang Q, et al. Association of a Cystatin $\mathrm{C}$ Gene Variant With Cystatin C Levels, CKD, and risk of incident cardiovascular disease and mortality. Am $J$ Kidney Dis. 2014;63:16-22. doi:10.1053/j.ajkd.2013.06.015

18. Satoh-Asahara N, Suganami T, Majima T, et al. Urinary Cystatin $\mathrm{C}$ as a potential risk marker for cardiovascular disease and chronic kidney disease in patients with obesity and metabolic syndrome. Clin J Am Society Nephrol Cjasn. 2011;6(2):265. doi:10.2215/ CJN.04830610

19. Oh KW. Cystatin C is a valuable marker for predicting future cardiovascular diseases in type 2 diabetic patients. Korean Diabetes $J$. 2008;32(6):488-497. doi:10.4093/kdj.2008.32.6.474 
20. Chawla R, Madhu SV, Makkar BM, et al. RSSDI-ESI Clinical practice recommendations for the management of type 2 diabetes mellitus 2020. Int J Diabetes Dev Ctries. 2020;40(Suppl):1.

21. Levey AS, Stevens LA, Schmid $\mathrm{CH}$, et al. A new equation to estimate glomerular filtration rate. Ann Intern Med. 2009;150 (9):604-612. doi:10.7326/0003-4819-150-9-200905050-00006

22. Radloff LS. The CES-D Scale: a self-report depression scale for research in the general population. Appl Psychol Meas. 1977;1 (3):385-401. doi:10.1177/014662167700100306

23. Karim J, Weisz R, Bibi Z, et al. Validation of the eight-item Center for Epidemiologic Studies Depression Scale (CES-D) among older adults. Current Psychol. 2015;34(4):681-692. doi:10.1007/s12144-014-9281-y

24. Pomeshkina S, Ardashova NY, Lebedeva NB, et al. Influence of type 2 diabetes and depression on subclinical inflammation in patients with myocardial infarction. Eur Heart J. suppl_1:P1338-P1338.

25. Baune SBT. Depression and type 2 diabetes: inflammatory mechanisms of a psychoneuroendocrine co-morbidity. Neurosci Biobehav Rev. 2012.

26. Bdescu SV, Ttaru C, Kobylinska L, et al. The association between diabetes mellitus and depression. J Med Life. 2016;9:2.

27. Kilic T, Oner G, Ural E, et al. Comparison of the long-term prognostic value of Cystatin $\mathrm{C}$ to other indicators of renal function, markers of inflammation and systolic dysfunction among patients with acute coronary syndrome. Atherosclerosis. 2009;207 (2):552-558. doi:10.1016/j.atherosclerosis.2009.05.015

28. Okura T, Jotoku, Jotoku M, et al. Association between cystatin C and inflammation in patients with essential hypertension. Clin Exp Nephrol. 2010;14:584-588. doi:10.1007/s10157-010-0334-8

29. Lee SH, Park SA, Ko SH, et al. Insulin resistance and inflammation may have an additional role in the link between cystatin $\mathrm{C}$ and cardiovascular disease in type 2 diabetes mellitus patients. Metabolism. 2010;59(2):241-246. doi:10.1016/j.metabol.2009.07.019
30. Schanz M, Pannes D, Dippon J, et al. The influence of thyroid function, inflammation, and obesity on risk prediction of acute kidney injury by cystatin $\mathrm{c}$ in the emergency department. Kidney Blood Press Res. 2016;41(5):604. doi:10.1159/000447929

31. Lertnawapan R, Bian A, Rho Y, et al. Cystatin C is associated with inflammation but not atherosclerosis in systemic lupus erythematosus. Lupus. 2012;21(3):279. doi:10.1177/0961203311425527

32. $\mathrm{Xu} \mathrm{Y,} \mathrm{Schnorrer} \mathrm{P,} \mathrm{Proietto} \mathrm{A,} \mathrm{et} \mathrm{al.} \mathrm{IL-10} \mathrm{controls} \mathrm{cystatin}$ $\mathrm{C}$ synthesis and blood concentration in response to inflammation through regulation of IFN regulatory factor 8 expression. J Immunol. 2011;186(6):3666-3673. doi:10.4049/jimmunol.1001934

33. Robakis TK, Aasly L, Williams KE, et al. Roles of inflammation and depression in the development of gestational diabetes. other. 2017;4:4.

34. Gregory JM. Inflammation and depression in patients with autoimmune disease, diabetes, and obesity. 2018;377-392.

35. Dona AC, Delouize AM, Eick G, et al. Inflammation and central adiposity as mediators of depression and uncontrolled diabetes in the study on global AGEing and adult health (SAGE). Am J Human Biol. 2020;32:6. doi:10.1002/ajhb.23413

36. Zahn D, Herpertz S, Albus C, et al. hs-CRP predicts improvement in depression in patients with type 1 diabetes and major depression undergoing depression treatment: results from the Diabetes and Depression (DAD) Study. Diab Care. 2017;39(10):e171. doi: $10.2337 / \mathrm{dc} 16-0710$

\section{Publish your work in this journal}

Diabetes, Metabolic Syndrome and Obesity: Targets and Therapy is an international, peer-reviewed open-access journal committed to the rapid publication of the latest laboratory and clinical findings in the fields of diabetes, metabolic syndrome and obesity research. Original research, review, case reports, hypothesis formation, expert opinion and commentaries are all considered for publication. The manuscript management system is completely online and includes a very quick and fair peer-review system, which is all easy to use. Visit http://www.dovepress.com/testimonials.php to read real quotes from published authors. 